\title{
Suitability of sheet bending modelling techniques in CAPP applications
}

\author{
A.H. Streppel, L.J. de Vin, J. Brinkman and H.J.J. Kals \\ Laboratory of Production Engineering, University of Twente, P.O. Box 217, 7500 AE Enschede, \\ the Netherlands
}

(Received February 26, 1992; accepted July 7, 1992)

\section{Industrial Summary}

The use of CNC machine tools, together with decreasing lot sizes and stricter tolerance prescriptions, has led to changes in sheet-metal part manufacturing. In this paper, problems introduced by the difference between the actual material behaviour and the results obtained from analytical models and FEM simulations are discussed against the background of the required predictable accuracy in small-batch part manufacturing and FMS environments. The topics are limited to those relevant to bending along straight lines. Applications of analytical and empirical models in the area of CAPP and adaptive control are discussed. Process planning for sheet bending, however, should not be treated as an individual task but must be related to other process-planning tasks. An integrated CAPP system for sheet metal, PART-S, is introduced briefly.

\section{Introduction}

Over the past few years, an increased effort has been put into research in the sheet-metal area, due partly to the introduction of CNC machine tools for sheetmetal manufacturing processes. Generally simultaneous with this development, customer demands changed, leading to decreased lot sizes, increased product variety and stricter tolerance prescriptions. As a result, a better and predictable process accuracy is required nowadays and test products should no longer be needed. This has led to research both on more traditional processes such as punching and bending and on relatively new processes such as laser cutting and laser welding. Furthermore, the workload for process-planning departments has increased (as has previously been the case for machining operations). Thus, the need for CAPP systems arises also in the sheet-metal area.

Correspondence to: L.J. de Vin, Laboratory of Production Engineering, University of Twente, P.O. Box 217, 7500 AE Enschede, the Netherlands. 
The overall accuracy of a sheet-metal product depends on the subsequent operations it undergoes, the bending process having a profound influence on this accuracy. With respect to the bending process, closed-die bending used to be the most common way of bending along straight lines. However, attention has moved towards air-bending, as this is a more flexible method allowing different products (with respect to angles, sheet thickness or material) to be bent without the need for frequent tool changes. Calculation of the correct punch displacement, however, is not easy. Many effects influence the springback and, consequently, the required punch displacement. Although most of these effects have been known for a long time already, their influence is not always easy to predict. Together with the increased accuracy demands, this is one of the reasons for the increased research effort on the bending process. Apart from analytical and experimental work, FEM simulations are used and attention has moved from description of individual effects towards the calculation of punch displacement and adaptive control.

In order to benefit fully from technological know-how, the bending process must not be treated as a separate operation. Process planning for bending must be imbedded in an integrated CAPP system, such a system including the selection of equipment, the determination of the bending sequence and instructions for positioning. A systematic analysis of tolerance prescriptions must be performed initially. Individual bending operations may be selected using manufacturing rules in combination with the accuracy of preceding and following operations (such as laser cutting, punching, laser welding and other bending). Finally, there is a strong relationship with planning aspects since, in small-batch manufacturing, different products are nested in one single sheet.

PART.S, a sheet metal CAPP system currently under development at the Laboratory of Production Engineering, offers this required integrated functionality. After completion, it may be used in CNC and FMS environments producing sheet-metal components in small batches within a thickness range of 0.5 to $5 \mathrm{~mm}$.

\section{Material behaviour}

Material behaviour is an important aspect in the understanding of the bending process. There are several models to describe the material behaviour, but some phenomena, however, are difficult to model.

\subsection{Modelled behaviour}

The behaviour of a material is described primarily by its stress-strain relationship, graphical representation of relationships being shown in Fig. 1. Two areas can be distinguished: an elastic region and a plastic region. The stress-strain diagram shown in Fig. 1 (a) represents a rigid-ideal plastic material. There is no work hardening nor an elastic region. A simple model like this may be used as a reference to describe the effects of an elastic region or work 

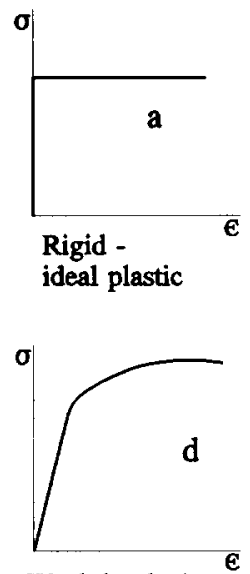

Work hardening according to Nadai
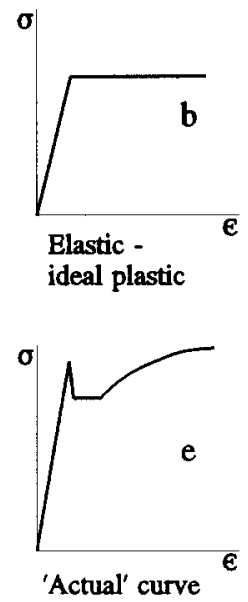
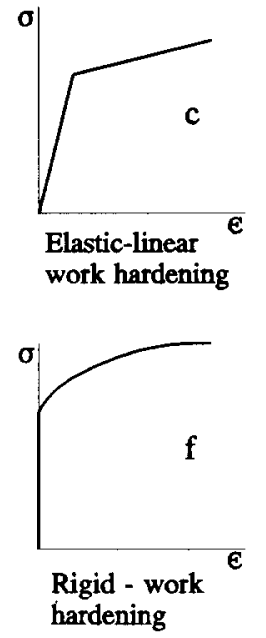

Fig. 1. Stress-strain diagrams.

hardening. A material without work hardening, but with an elastic region, is represented by the stress-strain diagram in Fig. 1(b). This model may be used, for instance, for rough estimations of spring-back.

Figures 1(c) and 1 (d) are examples of stress-strain diagrams with work hardening. Linear work-hardening is represented by the diagram in Fig. 1(c), whereas work hardening according to Nadai is shown in Fig. 1(d). These stress-strain relationships are those used most commonly to describe the material behaviour in analytical models. Some materials show an area without work hardening at the beginning of the plastic region, as in Fig. 1(e), this effect usually being neglected in analytical models. Material behaviour without an elastic region but with work hardening is illustrated by Fig. 1(f), such stress--strain relationships being useful for applications for which elastic effects are less important, for instance in the prediction of process forces.

\subsection{Actual material behaviour}

One of the biggest problems in the calculation of process parameters is that it is difficult to determine the values of the relevant material parameters. The most common experiment to determine these material parameters is the uniaxial tensile test. This test has two disadvantages: there is no plane-strain situation, and the deformation ratio occurring in sheet bending is usually larger than the maximum ratio in a uni-axial tensile test. The Miyauchi shear test allows larger deformation ratios, but is used less commonly [1]. The value of Young's modulus of a material is usually assumed to be fixed: however, Young's modulus may decrease substantially (some 16\%) under deformation [2] and as a result, the spring-back increases. 
In general, the flow curve will depend on the strain path followed [3]. For a total reversal of the strain path, the Bauschinger effect occurs. As a sheet is produced by rolling, this phenomenon occurs at the compressive side during bending. Anisotropic material behaviour is caused also by the production process of the sheet. Anisotropy is expressed by means of the plastic strain ratio $R$ [4]. More important in sheet bending is the phenomenon that the ratio $R$ may differ in different directions: the so-called planar anisotropy. As a result, the material behaviour depends on the orientation of the bending line with respect to the rolling direction of the sheet.

The phenomena mentioned above may differ in importance and do not always have a profound influence on the bending process. However, it may sometimes prove difficult to determine the correct values of the relevant material parameters. This especially applies to production environments, due to the lack of testing equipment and time.

\section{Theory of sheet bending}

\subsection{Assumptions}

The bending process was first subjected to a thorough investigation by Ludwik [5]. The assumptions made by him and later adopted by others [6,7] are as follows:

(i) the thickness and width of the sheet are uniform throughout the sheet and remain constant during the bending process. The sheet is bent by a pure moment, therefore the bending line is a circular arc;

(ii) the sheet has a large width/thickness ratio, so edge deformations are negligible and a plane state of strain exists;

(iii) the material is homogeneous and isotropic and there are no residual stresses present prior to bending;

(iv) plane sections normal to the sheet surface and parallel to the bend axis remain plane;

(v) the sheet consists of many thin independent layers. Stresses perpendicular to the sheet surface and stresses parallel to the bend axis are neglected;

(vi) the stress-strain curves of a workpiece in the tensile and compressive regions are symmetric with respect to the origin;

(vii) from assumptions (i)-(vi) and volume constancy, it follows that the sheet thickness remains constant during the bending process.

To satisfy these conditions it is required that the width/thickness ratio of the sheet and the bending radius/thickness ratio are sufficiently large.

\subsection{Bending moment and process force}

The strains and stresses in the bending plane may be analyzed (Fig. 2) under the conditions mentioned in Section 3.1. From assumptions (i) to (iv) it follows that $\varepsilon_{z}=0$ and $\sigma_{x}, \sigma_{y}$ and $\sigma_{z}$ are the principal stresses, whilst from assumption 

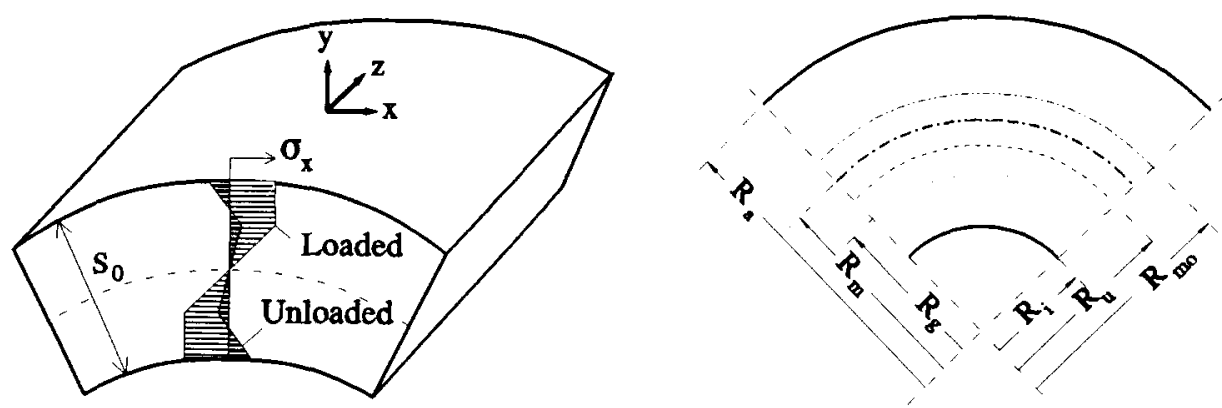

Fig. 2. Stresses and layers in a sheet.

(v) it follows that $\sigma_{y}$ and $\sigma_{z}$ may be neglected. The bending moment $M_{\mathrm{b}}$ (with $s_{0}$ as the sheet thickness and $b$ as the width) may be calculated using:

$M_{\mathrm{b}}=2 \int_{0}^{s_{0} / 2} \sigma_{x}(y) b y \mathrm{~d} y$

The value of this moment depends on the stress strain relationship used. In calculations, sometimes a unit bending moment (which is the bending moment required to bend a sheet with a width and a thickness of $1 \mathrm{~mm}$ ) is used. The value of this unit bending moment depends on the stress-strain relationship and the strain in the outer fibre (the ratio $s_{0} / 2 R_{\mathrm{m}}=\varepsilon_{x_{\mathrm{a}}}$ ). Several expressions for bending moment and process force have been derived, based on different stress-strain relationships [8-10]. Usually, friction between the workpiece and the die is taken into account.

The process force is usually calculated based on the stress distribution in the bending zone. The bending moment is calculated, and as a result the reaction forces between the sheet and the die may be determined. For this purpose, an approximation of the bending geometry based on a rigid-plastic model is used often (Figs. 1 (a) and 3). Thus, a process force-punch displacement diagram may be determined taking into account the friction between the sheet and the die, an example of such a diagram being given in Fig. 4. At some point, the process becomes that of closed-die bending and the process force rises very quickly [11,12]. The final stage of closed-die bending is shown in Fig. 3 , at the lower right. Referring to Fig. 3 and neglecting the friction force, the process force may be calculated from

$$
F_{\mathrm{p}}=\frac{2 M_{\mathrm{b}}}{L \cos ^{2}(\alpha / 2)} \quad L \cos (\alpha / 2)=H / 2
$$

The right-hand side equation gives an approximation which is valid only if the die opening $H$ is large with respect to radii $R_{\mathrm{p}}$ and $R_{\mathrm{d}}$. Nevertheless, $H$ is considered often to be a process parameter although the length of the free section $L$ is more important. The influence of friction is taken into account easily by modifying the process force equation. 


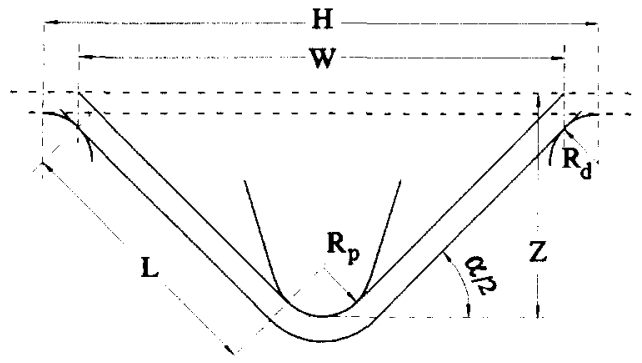

Geometry (rigid-plastic)

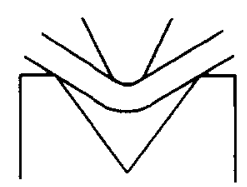

Air-bending

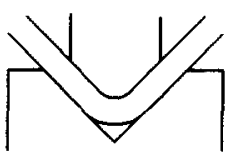

Closed-die bending

Fig. 3. Brake forming.

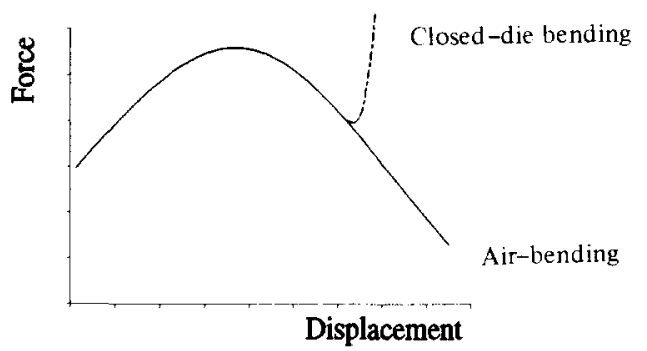

Fig. 4. Example of a process force-punch displacement diagram.

\subsection{Spring-back}

To describe spring-back using analytical models, the bending moment is usually split into an elastic and a plastic contribution: $M_{\mathrm{b}}=M_{\mathrm{el}}+M_{\mathrm{pl}}$. Based on elastic-ideal plastic material, the stresses before and after spring-back are illustrated in Fig. 2. Other stress-strain relationships lead to different residual stresses and different spring-back angles [13,14]. However, common trends are that spring-back increases with: (i) decreasing sheet thickness; (ii) increasing yield strength; (iii) increasing bending radius; and (iv) decreasing Young's modulus. The spring-back is expressed often by the ratio $K$, being the ratio between the bend angles after and before spring-back $(K \leqslant 1)$.

The bend radius is often assumed to be uniform throughout the bending zone (assumption (i)). Other parts of the sheet are assumed to remain flat after spring-back (so called circular-straight models). However, for accurate calculation of product angles, both before and after spring-back, these assumptions are not valid, since the bend radius varies along the bending zone $[9,15]$. As a result, bend angles before spring-back (and, consequently, after spring-back) are not equal to those calculated from a circular-straight model.

\subsection{Length of the bent section}

At the beginning of the bending process, a neutral layer exists at the middle of the sheet, but this situation changes during the bending process. The layers 
with strains equal to zero and stresses equal to zero no longer coincide $[6,8,9,12]$. There are several layers, with changing positions as the bending process continues (Fig. 2). The material between the outer radius $R_{\mathrm{a}}$ and the initial middle layer $R_{\mathrm{m} 0}$ has been stretched. The material between $R_{\mathrm{m} 0}$ and $R_{\mathrm{g}}$ has first been compressed and later been stretched: the Bauschinger effect thus ocsurs in this zone. The total deformation of the layer indicated by $R_{\mathrm{u}}$ is equal to zero. The material between $R_{\mathrm{g}}$ and $R_{\mathrm{i}}$ has been compressed. Assuming that the sheet thickness is constant, the length of the layer with total deformation equal to zero (thus, the length of the unfolded bend) may be calculated. The relative difference in length $\mathrm{d} L / L_{\mathrm{m}}$ ( $L_{\mathrm{m}}$ is the length of the middle layer) increases as the bending radius-sheet thickness ratio, $R_{\mathrm{m}} / s$, decreases. However, the sheet thickness does not remain constant but decreases if work hardening occurs $[9,16]$. This effect is stronger for larger work hardening and smaller $R_{\mathrm{m}} / \mathrm{s}$ ratios. As a result of this complication, calculation of the unfolded sheet length is usually based on experimental data.

\section{FEM approach}

In the development of theoretical models for the sheet-bending process, several assumptions have been made (see Section 3.1), but in industrial sheetbending processes these conditions are not satisfied. In fact, the process is started by shear forces instead of by a pure bending moment. Thus, a shear deformation will occur over the sheet thickness. Because of this, the bending moment and the bend radius are not constant and it is not possible to predict the geometry of the bent sheet sufficiently accurately with simple analytical models based on theory employing the assumptions mentioned above.

FEM simulation is not suitable for use in a CAPP system, due to the computing time needed. However, FEM simulation is a very useful tool in determining the influence of several parameters. Comparing the results of such simulations with theory and experiment, it could be possible to develop a mathematical model for sheet bending that can be used in a CAPP system.

In 1980 Kobayashi and Oh [17] analyzed sheet bending as a bulk-deformation process. They assumed a process without friction and used two material models: a rigid-plastic and an elastic-plastic. To calculate the spring-back angle in the case of a rigid-plastic material, unloading was also done according to elastic-plastic behaviour. The results of both methods correspond. Because of the lesser computing time, these authors prefer the rigid-plastic material model. Other research on FEM simulation has been done by Makinouchi [18], who also neglects friction. Instead of using a standard program (available on the market) he developed a special-purpose program for bending of (laminate) sheet metal, which gives the advantage of shorter computing time.

It is common practice to use a fine mesh in the sheet-punch contact area. If friction between the sheet and the die is taken into account, a fine mesh is needed for the sheet-die contact area (Fig. 5) also. This friction is especially 

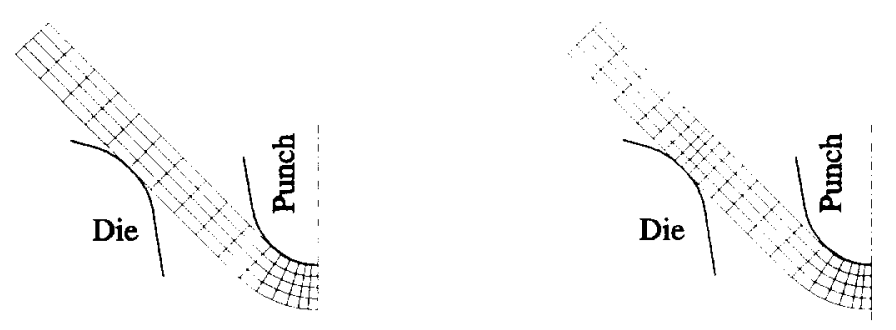

Fig. 5. Examples of FEM meshes.

important if small die-openings, according to industrial practice, are used. In this case, the contact forces are very high.

More work on FEM simulation has been reported, but the results are not always applicable for the air-bending of sheet: the ratio of the die opening to the sheet thickness does not resemble industrial practice, friction is not taken into account, or the ratio of the sheet width to the thickness is too small.

\section{Experimental verification}

\subsection{Bending theory}

Bending equipment consisting of three cylindrical rolls was used by Ludwik [5], who found a difference of $10 \%$ in bending moment between theoretical and experimental results: this is probably due to the sheets not being bent by a pure bending moment. Wolter [9] used special equipment which allowed him to bend sheets with a pure moment, finding good correspondence between the bending moment needed in his experiments and the theoretical bending moment. This is remarkable, as he neglected the stress $\sigma_{z}$, whereas, according to Tresca and von Mises, $\sigma_{z}$ is equal to $\left(\sigma_{y}+\sigma_{x}\right) / 2$. Often a unit bending moment is calculated using the following equation:

$M_{\mathrm{u}}=\frac{M_{\mathrm{b}}}{b s_{0}^{2}}$

This equation is based on the assumption that the strain distribution is symmetrical and linear [19]: for small bend radii especially, this is not the case [20]. As a result, different experiments may lead to different unit bending moments for the same material. A second assumption made is that the shear force has no influence on the unit bending moment.

\subsection{Plane strain}

Tozawa [21] has done work in the field of the behaviour of steel under different stress conditions and anisotropy. An example of the difference between stress-strain curves for a uni-axial and a plane-strain situation is given in Fig. 6, left. 

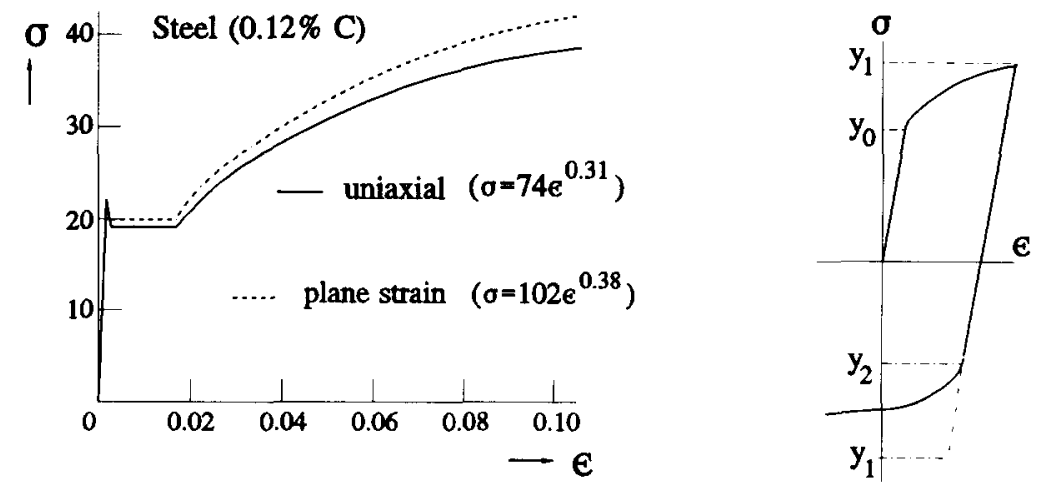

Fig. 6. Plane strain (left) and the Bauschinger effect (right).

\subsection{Planar anisotropy}

Other experiments by Tozawa [22] concentrated on planar anisotropy, which is substantial for steel under compressive or tensile stress. However, a direct relationship between planar anisotropy under these conditions and during bending cannot be given, due to bending taking place under plane-strain conditions. As a result of planar anisotropy, differences of up to $16 \%$ may be found if the unit bending moment is determined for bending lines with different orientations with respect to the rolling direction of a sheet.

\subsection{The Bauschinger effect}

The Bauschinger effect has been investigated thoroughly. However, little research has been concentrated on this phenomenon in relation to sheet bending. A flow curve of a material with the Bauschinger effect is shown in Fig. 6 (right), the material displaying a mixed behaviour of isotropic yielding and kinematic hardening. Rolfe [23] has investigated the Bauschinger effect using test-specimens taken from a bent sheet of $2 \frac{1}{2}^{\prime \prime}$ thickness $(63.5 \mathrm{~mm})$ with different orientations (transverse and longitudinal) and from both compressive and tensile regions. His experiments showed a substantial Bauschinger effect for cold-rolled steel: the yield strength in the compressive region may be as much as $30 \%$ lower than the yield strength in the tensile region.

Weinmann [24] used thin sheet $(0.86 \mathrm{~mm})$ which he bent cyclically with a pure moment. The Bauschinger effect resulted in a reducing unit bending moment every time the bending moment was reversed. The Bauschinger effect factor (BEF) was used to quantify the effect, this factor being defined as (see also Fig. 6, right):

$\mathrm{BEF}=\frac{Y_{1}-\left|Y_{2}\right|}{Y_{1}}$

In general, pre-strained material has a larger BEF for the first cycle but reaches a stable value after a few cycles. 


\subsection{Sheet length}

The length of the bending zone (that is, the length of the layer indicated by $R_{\mathrm{u}}$ in Fig. 2) is important for the calculation of blank dimensions. As has been mentioned before, there are no suitable analytical models to calculate this length when the bend radii are small (the initial assumptions made in Section 3.1 are no longer satisfied), which is the case for many industrial products. The presence of shear forces and contact forces are additional complications. Furthermore, it has been mentioned that the curvature of the bending zone is not constant. Two qualitative effects may be mentioned: (1) when the geometry differs more strongly from a circular arc, the length of the bending zone is smaller, and (2) when the contact forces are lower, the radius $R_{\mathrm{u}}$ becomes larger and thus the length of the bending zone is larger.

The length of the bending zone is usually calculated according to the ASM or DIN standards $[1,25]$. Both standards make use of equations with a correction factor based on experimental data.

The ASM standard uses the following equations for steel:

$L=\left(t p+r_{\mathrm{i}}\right) 0.01745 \beta$

with:

$p=\frac{r_{\mathrm{i}}}{t} 0.04+0.3 \quad$ for $\frac{r_{\mathrm{i}}}{t}<1$

$p=\left(\frac{r_{\mathrm{i}}}{t}-1.0\right) 0.06+0.34$ for $\quad \frac{r_{\mathrm{i}}}{t} \geqslant 1$

with $\beta$ in degrees and $r_{\mathrm{i}}, t$ and $L$ as shown in Fig. 7, left. The DIN standard uses the following equations for steel:

$L=a+b+v, \quad v=\pi\left(\frac{180^{\circ}-\alpha}{180^{\circ}}\right)\left(r_{\mathrm{i}}+\frac{s}{2} k\right)-2\left(r_{\mathrm{i}}+s\right)$

$k=0.65+0.5 \log \left(\frac{r_{\mathrm{i}}}{s}\right)$, note that $r_{\mathrm{u}}=r_{\mathrm{i}}+\frac{k s}{2}$

with $\alpha$ in degrees and $a, b, r_{\mathrm{i}}, s$ as shown in Fig. 7, right. $L$ is the total length of the (unfolded) sheet.

It is suggested that $r_{\mathrm{i}}=r_{\mathrm{m}}$ if $r_{\mathrm{i}} / s$ is sufficiently large. According to DIN this is the case for a ratio of 5 , whereas according to the ASM equation a ratio of 3.67 is sufficient.

Jahnke [26] has carried out research in the same field. For $\alpha=90^{\circ}$, he developed a simpler equation to calculate the length $v$ in the DIN equation: $v=\left(0.429 r_{\mathrm{i}}-1.476 s\right)$

\subsection{Minimum bend radius}

The minimum bend-radius is limited by the strain in the outer fibres. Fracture occurs when this strain is about 1.5 times the fracture strain of a uni-axial 

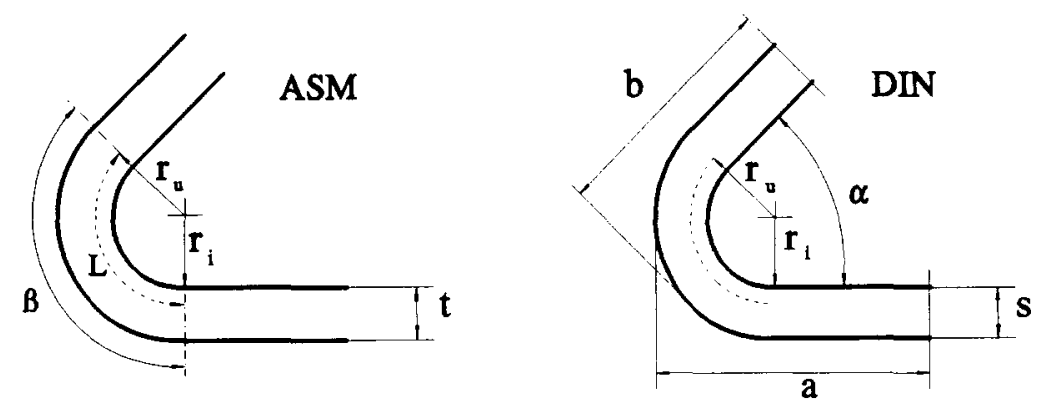

Fig. 7. Sheet length according to ASM and DIN.

tension test, as was found by Jahnke [26]. For smaller bend angles and low width/thickness ratios a larger strain is possible. When the bend is parallel to the rolling direction or the edge quality is poor, fracture occurs at a lower strain. To avoid initial fractures, the strain should not exceed 20 to $25 \%$, as may be calculated from various standards such as DIN 6935 and 9003 [25,27]. However, even for smaller deformations the surface roughness will increase, as indicated by Dautzenberg [28]. The limits mentioned should act as design rules.

\subsection{Spring-back}

Spring-back is expressed often as the spring-back factor $K$, which equals $1+\Delta \alpha / \alpha$ ( $\alpha$ is the bend angle, $\Delta \alpha$ the spring-back). However, $K$ is not a material parameter but depends on the bend angle and the ratio between the sheet thickness and the bend radius. Schwark [13] and Föppl [15] found good correspondence between average experimental spring-back and calculated springback, the actual spring-back, however, showing a large variation. It should be realised also that the prediction of spring-back itself is not sufficient for CAPP applications. For CAPP, correct prediction of the punch displacement is required in order to obtain the required product angle.

\subsection{Batch variation}

It has been mentioned above that the actual spring-back may vary considerably. This may be caused by variation in sheet thickness and yield strength. A thickness variation of 15 to $20 \%$ is allowed for thin cold-rolled sheet, according to DIN 1541 [29]. Even within one batch or one sheet, differences in sheet thickness may occur. Furthermore, the yield strength may show variations of $20 \%$, as indicated in DIN 1623 [30]. Both the thickness and the yield strength have a profound influence on the spring-back. Thus, it will be understood that the control of spring-back is a serious problem.

\subsection{Contact behaviour}

Spring-back is influenced also by the distribution of the contact forces in the wrap-around zone. In some cases, however, there is no wrap-around zone. In 
the case of a small tool-radius in combination with a large die-opening, the radius may be greater than the tool radius (Fig. 8, left). Under the influence of shear forces, the sheet may lose contact with the centre of the punch, as shown in Fig. 8, right.

In the case of air-bending, it will be understood that the product angle and length of the bending zone will be influenced by these effects. When the final state of the bending process is that of closed-die bending, these effects may result in an unpredictable (or even negative) spring-back.

\section{In-process control}

In order to achieve the required product accuracy, it is necessary to ensure that bend angles stay within certain - usually narrow - tolerances.

\subsection{Processes}

When closed-die bending is selected as the manufacturing process, relatively large variations of the product angle must be allowed: this is caused by the angle before spring-back being determined uniquely by the selected tools. As has been seen, spring-back may vary considerably due to variations in sheet thickness and yield strength. Thus, the angle obtained after spring-back will vary accordingly.

When air-bending is selected and the variation in sheet thickness is not taken into account, the deviation will be even larger, since the actual punch displacement differs from the calculated value (which is based on the nominal sheet thickness). However, when it is possible to control the spring-back by adapting the punch displacement to the actual process conditions, then two important results are achieved. The first result is an adequate control of the deviation of the bend angle, regardless of variations in sheet thickness and yield strength, whilst the second result is that one set of tools can be used for the manufacturing of different bend angles for a wide range of sheet thicknesses and material qualities.

\subsection{Load-unload method}

The load-unload method is based on the measurement of the angle obtained under loading and the spring-back angle after partial unloading. The equipment used is shown in Fig. 9. Instead of a conventional die, a die consisting of
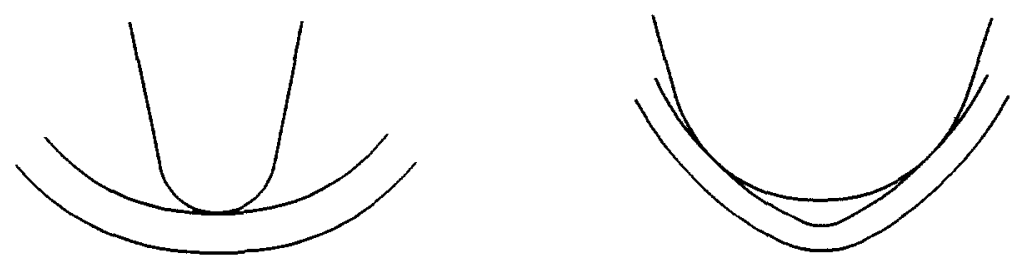

Fig. 8. Special contact behaviour. 


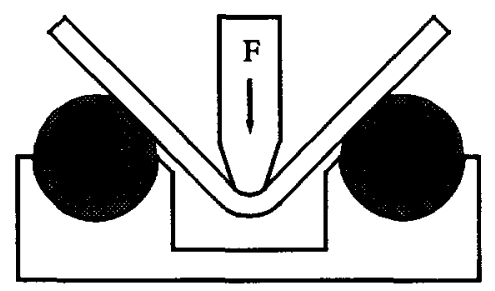

Fig. 9. Special die.

rolls with flat faces is used. After the first bending step, the bent angle is measured. Secondly, the bending force is reduced to $50 \%$ : the spring-back is measured and the required angle under loading is calculated. Finally, the product is bent until this latter angle is reached. This method has been investigated by von Finckenstein [31], who indicates that it is important to know the actual relationship between force-reduction and spring-back, which is non-linear due to friction. A disadvantage of this method is that tool-costs are high and that the application range is limited. The method is used in industry for the bending of long members.

\subsection{Force-displacement method}

This method is based on the measurement of the force punch displacement curve. Using this curve, it is possible to determine the sheet thickness and the mechanical properties. Determination of the sheet thickness is relatively straight-forward, as the thickness can be calculated from the displacement at the point where the force starts to rise. In order to determine the mechanical properties of the sheet, comparison with experimental data or a model is needed. Stelson $[14,32]$ has analyzed this method, finding that the results may be very satisfactory (angles to within $1^{\circ}$ ) if a realistic model is used and enough data on the force-displacement curve are collected. Over-bending of the product tends to occur when work hardening is not included in the model, due to the theoretical geometry and the actual geometry of the sheet near the wraparound zone differing: as a result, the product is bent more than is intended. The larger spring-back does not compensate for this overbend error, especially when a small die-opening is used. For large die-openings, the error may be over-compensated for. As for the problem of collecting sufficient data, Stelson indicates that this may be a problem for large product angles. It may be expected that this problem also arises when small die-openings are used (in this case the punch displacement is low also). Thus, in-process control has two aspects: the modelling problem and the identification problem.

\subsection{Alternative methods}

An alternative method is the measurement of both the angle and the force, provided that the right equipment is available for use in an industrial environment: in this case, the sheet thickness can be calculated. The sheet is bent until 
a given (safe) angle is reached. Measurement of the force is needed to calculate the spring-back and the required angle under loading. The amount of data that must be collected is relatively low.

When a simple, uniform bending test is available, an even simpler means of in-process control is possible. Such a test should require simple equipment and the amount of data to be collected is low. In this case, the test can be performed in a production environment using, for instance, two or three test-pieces taken from each sheet. The results of this test are characteristic for the mechanical properties of the sheet. For each bend, only the sheet thickness must be determined in-process (the thickness can vary throughout a sheet).

\section{Computer aided process planning (CAPP)}

\subsection{Process planning tasks}

Process-planning tasks related to bending include the selection of equipment (tools and press brakes), the calculation of unfolded blank dimensions and the required punch displacement, and the determination of the bending sequence. These tasks are interrelated in many ways, one obvious relationship being the relationship between the die opening and the required punch displacement. The die opening also influences the contact behaviour of the sheet and the required process force. Another important aspect is the relationship between the bending sequence and the tools to be used [33]. To avoid collisions, the tools selected must be within certain attribute ranges.

The determination of the bending sequence is related also to accuracy aspects. The actual accuracy required of bend angles is mostly not indicated explicitly by tolerances on angles, but must be calculated from tolerances indicated on linear dimensions, such as the distance between holes. In general, the resulting admissible tolerance in bending depends also on the bending sequence and the positioning strategy. Thus, by selecting the right bending sequence and suitable positioning edges, the admissible errors in bending may be increased or are, at least, not unnecessarily restricted. This can, for instance, result in more products being able to be bent on press brakes with standard equipment, thus relieving the workload for press brakes equipped for in-process control.

\subsection{PART:S: An integrated process-planning system}

As has been indicated, process planning for sheet-metal products is more than just calculation of the required punch displacement (although this is indeed an important task). An integrated process-planning system currently under development at the authors' Laboratory is PART-S [34]. Its architecture (Fig. 10) is based on the architecture of PART, which is a process-planning system for prismatic parts, presently in use in industry [35]. The processplanning functions, however, are partly different. Characteristic for sheet metal are the functions related to the generation of unfolded blank sizes and nesting. The unfolding function is needed because the output from the design 


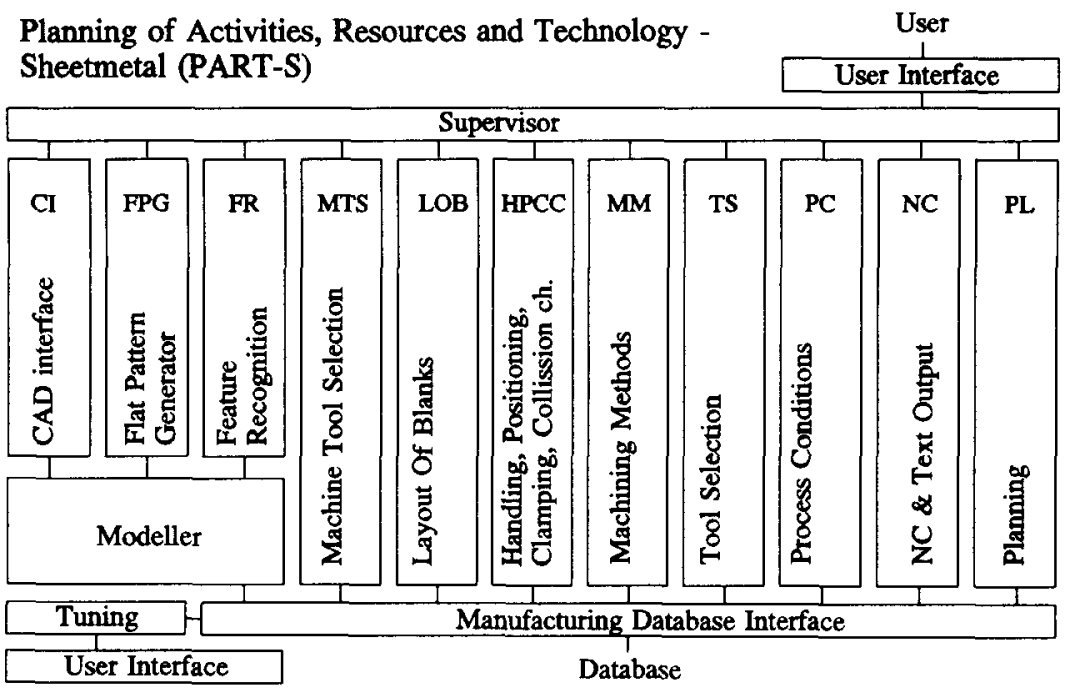

Fig. 10. PART-S reference diagram.

process will, in general, not include a model of the blank. Nesting functions are required since, in small-batch production of sheet-metal products, many (often different) products are nested in one sheet. Nesting is not just a geometrical problem but has strong relationships with planning aspects.

PART.S offers fully automated facilities to analyze and manipulate boundary representation (B-rep) product models stored in the internal modeller. The process planning functions in PART-S are:

CI - CAD Interface. This module offers the possibility to exchange product models with external CAD systems via STEP or neutral file format.

FR - Feature recognition. This module extracts manufacturing features such as bending lines or flat-pattern features from the product model.

FPG - Flat-pattern generator. A module which generates the unfolded blank dimensions of the product and a B-rep representation of this blank.

MM - Selection of the machining methods. This function has to be performed at several levels, for instance the choice between punching/nibbling and laser cutting, but also the determination of punching sequences.

MTS - Selection of machine tools. The selection of machine tools is one of the first decisions one would like to make, but in general, this is not possible, as many factors influence this decision. In the case of a press brake for instance, the required back gauge facilities depend on decisions made later. The procedure is to reduce the number of candidate machine-tools stepwise.

PL - Capacity planning. Flexible manufacturing requires a relatively short planning horizon, which means that planning tasks such as handling priority of orders, the allocation of jobs and tools, and load balancing must be integrated with technical process-planning. 
LOB - Layout of blanks. This function is characteristic for the small-batch production of sheet-metal parts. In this type of production, usually several different products are nested in one sheet in order to reduce set-up times. Material utilization is a possible optimization goal. More important, however, is that machining methods for the nested products must correspond and that constraints imposed by planning aspects are taken into account.

HPCC - Determination of handling, positioning and clamping. Positioning and clamping for flat-pattern operations are relatively straight-forward. The determination of handling and positioning for bending operations, however, is one of the major tasks of a sheet-metal CAPP system. It includes evaluation and conversion of tolerances, evaluation and subsequently selection of candidate bending operations and positioning edges.

TS - Tool selection. The appropriate tool-attribute ranges are determined during method selection. Based on the availability of tools and common attribute ranges, the best-fitting tools are selected.

PC - Determination of process conditions. This can be relatively straightforward for punching and nibbling, but is complicated for bending, laser cutting and laser welding.

$\mathrm{NC}-\mathrm{NC}$ and text output. This module generates $\mathrm{NC}$ files and operator instructions.

Each module is subdivided in a number of so-called phases: independent programs communicating with each other via the database. The execution of a process-planning session is controlled by a scenario in which the execution sequence of the various phases is stored and is carried out by the supervisor. Scenarios are user definable. The use of scenarios allows users with different views on process planning to use PART-S in their own companytailored way.

\section{Concluding remarks}

As has been discussed, analytical models have some serious limitations. Under industrial conditions (small bend-radii, small die-openings) the assumptions needed for analytical description of the bending process are no longer valid. However, analytical models may support certain decisions in the process-planning phase, for instance, the estimation of required punch force or the extrapolation and interpolation of empirical data. In the case of in-process control, analytical models may be used to calculate a safe first-value for the punch displacement.

A typical application of empirical models is the calculation of the unfolded blank dimensions. Presently, these models do not take into account the influence of die opening, yield strength and the Bauschinger effect. To improve the accuracy of these models, further research on these topics is needed. A typical problem related to the use of empirical models for the calculation of the required punch displacement is the necessity to collect numerous data for different angles, tools, materials and sheet thicknesses. 
FEM simulation is not suitable for use in the process-planning phase, due to the computer time needed. Furthermore, when the computing time can be reduced, the necessity to have the actual material data available is still there. However, FEM simulation is a very useful tool to determine the influence of several parameters.

In-process control is an alternative to deal with variation of mechanical properties and sheet thickness. This approach, however, does not eliminate the necessity for both modelling and identification of material behaviour. It is not yet suitable for use in small-batch manufacturing with its typical wide range of product types. A suggestion for future research in this field is the development of an in-process control method requiring less data. In general, identification of material parameters is difficult. A simple test, to be performed in a production environment, should be developed to deal with this problem.

During process planning, many decisions have to be made, most of these decisions being interdependent. Thus, the need for integrated CAPP is evident. The required accuracy of bend angles is influenced by the selected bending sequence and positioning edges. Integration of different process-planning functions in one system allows this influence to be taken into account, which means that tolerances are not unnecessarily restricted and the use of a wider range of equipment (especially standard tools and press brakes) is possible. On the other hand, standard sequences and handling procedures can be used when the alternatives do not lead to substantial advantages.

\section{References}

[1] ASM, ASM-Metals Handbook, 9th ed., American Society for Metals, Metals Park, OH, Vol. 1, 1978, pp. 548-549, pp. 552-554; Vol. 8, 1985, pp. 118-129, pp. 552-558, pp. 564-569; Vol. 14,1988 , p. 12 , pp. $523-525$, p. 531 , pp. $541-543$, p. 552 , p. 629 , p. 646 , pp. $656-657$, pp. $762-763$, p. 776 , p. 792 , p. 794 , pp. $820-821$, p. 825 , p. 839 , p. 846 , pp. $880-881$, pp. 887-894, pp. 913-922.

[2] W. Lems, The change of Young's modulus after deformation at low temperature and its recovery, Ph.D. Dissertation, Delft, 1963.

[3] W.H. Sillekens, J.H. Dautzenberg and J.A.G. Kals, Strain path dependence of flow curves, Ann. CIRP, 40(1) (1991) 255-258.

[4] ASTM, Mechanical testing, Standard test method for plastic strain ratio $R$ for sheet metal, Annual Book of ASTM Standards, Section 3, Vol. 03.01, Des. E 517-81, 1987.

[5] P. Ludwik, Technologische Studie über Blechbiegung (Sonderabdruck aus den Technischen Blättern), Deutschen Polytechnischen Vereins, Böhmen, Prag, 1904.

[6] C. Bach, Elasticity and Strength, Springer, Berlin, 1934.

[7] A. Nadai, Plasticity of Materials, Springer, Berlin, 1927.

[8] R. Hill, The Mathematical Theory of Plasticity, Clarendon Press, Oxford, 1950.

[9] K.H. Wolter, Freies Biegen von Blechen, VDI-Forschungsheft Vol. 435, Ausgabe B, Bd. 18, Deutscher Ingenieur-Verlag, Düsseldorf, 1952.

[10] B. Zünkler, Rechnerische Erfassung der Vorgänge beim Biegen im V-förmigen Gesenk, Industrieanz., 73 (1966) 213-217.

[11] M. Hermann, M. Wilhelm and P. Keck, Process simulation of metal-forming processes: Some examples, Ann. CIRP, 40(1) (1991) 285-289.

[12] K. Lange, Blechumformung, Lehrbuch der Umformtechnik, Bd. 3, Springer, Berlin, 1975. 
[13] H.F. Schwark, Rückfederung an bildsam gebogenen Blechen, Ph.D. Dissertation, T.H. Hannover, 1952.

[14] A. Föppl, Vorlesungen über Technische Mechanik, Bd. 5, Leipzig, 1907, pp. 73-81.

[15] K.A. Stelson, Real time identification of workpiece-material characteristics from measurements during brakeforming, J. Eng. Ind., Trans. ASME, 105 (1983) 45-53.

[16] F. Proksa, Plastisches Biegen von Blechen, Stahlbau, 2 (1959) 29-36.

[17] S. Kobayashi and S.I. Oh, Finite elements analysis of plane-strain sheet bending, Int. $J$. Mech. Sci., 22 (1980) 583-595.

[18] A. Makinouchi, Elastic-plastic stress analysis of bending and hemming of sheet metal, in: N.B. Wang and S.C. Tang (Eds.), Computer-Modeling of Sheet Metal Forming Processes, Metallurgical Society, 1985, pp. 161-176.

[19] G. Oehler, Biegen, Carl Hanser, München, 1963.

[20] G. Nicklas, Das Biegen von Blechen an Hand einer Biegung in V-Gesenken (Ph.D. Dissertation), VDI, Düsseldorf, 1976.

[21] Y. Tozawa, Plastic deformation behavior under conditions of combined stress, in: D.P. Koistinen and N.M. Wang (Eds.), Mechanics of Sheet Metal Forming, Plenum Press, London, 1978, pp. 81-110.

[22] Y. Tozawa, N. Kanetake, A. Honda and H. Kudo, Prediction of the bending moment of predeformed sheet from uniaxial test results, Ann. CIRP, 29(1) (1980) 163-166.

[23] S.T. Rolfe, R.P. Haak and J.H. Gross, Effect of state-of-stress and yield criterion on the Bauschinger effect, J. Basic Eng., Trans. ASME, (September 1968) 403-408.

[24] K.J. Weinmann, A.H. Rosenberger, L.R. Sanchez and B.T. von Turkovich, The Bauschinger effect of sheet metal under cyclic reverse pure bending, Ann. CIRP, 37(1) (1988) 289-293.

[25] DIN 6935, Kaltbiegen von Flacherzeugnissen aus Stahl, Biegehalbmesser - kleinste Schenkellänge - zulässige Abweichungen der Winkelstellungen - Berechnung der gestreckten Längen, 1975.

[26] H. Jahnke, R. Retzke and W. Weber, Fertigungstechnik, Umformen und Schneiden, VEB, Berlin, 1971.

[27] DIN 9003, Biegen von Blechen, Platten und Bändern aus Stahl und hochwarmfesten Legierungen, Biegehalbmesser, Konstruktions-richtlinien, 1984.

[28] J.H. Dautzenberg and J.A.G. Kals, Surface roughness caused by metal forming, Ann. CIRP, 34(1) (1985), 477-479.

[29] DIN 1541, Kaltgewaltzes Breitband und Blech aus unlegierten Stählen, Masse, zulässige Mass- und Formabweichungen, 1975.

[30] DIN 1623, Kaltgewaltzes Band und Blech aus weichen unlegierten Stählen, Gütevorschriften, 1972.

[31] E. von Finckenstein and R. Rothstein, Gesenkbiegen von Blechen, Automatische Rückfederungskorrektur, Industrieanz., 24 (1988) 19-20.

[32] K.A. Stelson, An adaptive pressbrake control for strain hardening materials, J. Eng. Ind., Trans. ASME, 108 (1986) 127-132.

[33] M. Hoffmann, U. Geißler and M. Geiger, Computer-aided generation of bending sequences for die-bending machines, J. Mater. Process. Technol., 30 (1992) 1-12.

[34] L.J. de Vin, J. de Vries, A.H. Streppel and H.J.J. Kals, PART-S, a CAPP system for small batch manufacturing of sheet metal components, 24th CIRP Int. Seminar on Manufacturing Systems, Copenhagen, 1992, pp. 171-182.

[35] F.J.A.M. Houten, PART: A computer aided process planning system, Ph.D. Dissertation, Enschede, 1991. 